\title{
The AGS-Booster Complex for the g-2 Experiment and RHIC Injection*
}

\author{
Mitsuyoshi TANAKA and Yong Y. Lee \\ Brookhaven National Laboratory \\ Upton, NY 11973
}

\section{Abstract}

The delivery of a beam with characteristics appropriate for the g-2 muon storage ring and the filling of the RHIC heavy ion collider from the AGS main ring requires a new fast extracted beam (NewFEB) system. The NewFEB system must be capable of performing single bunch multiple extraction of a heavy ion beam, as well as a high intensity proton beam at a time interval of $25 \mathrm{~ms}$ up to 12 times per AGS cycle. The new system will consist of a fast multipulsing kicker and an ejector septum magnet with local orbit bumps.

\section{INTRODUCTION}

Since the present fast extraction beam (FEB) and single bunch extraction (SBE) systems [1] are no longer available for the post-Booster era, the NewFEB system [2] will serve as the AGS extraction system not just for the muon g-2 experiment [3] but also for RHIC [4] and the recently approved long baseline neutrino oscillation experiment [5] at AGS as well. The AGS Booster, recently completed, allows the AGS to accelerate heavy ions (HI) beyond $\mathrm{Si}^{28}$ up to $\mathrm{Au}^{197}$ and will increase the proton intensity in the AGS by a factor 4 [6].

For the g-2 experiment, which is now constructing a $14 \mathrm{~m}$ diameter superferric muon storage ring with $\mathrm{B}=1.5 \mathrm{~T}$ in order to improve the previous CERN measurement of the anomalous magnetic moment $\left(a_{\mu}\right)$ by a factor of 20 , NewFEB must meet the following requirements: (1) extract the bunched proton beam up to full energy and intensity to the new $\mathrm{V}$ target through the existing U-line for $3.1 \mathrm{GeV} / \mathrm{c}$ pion production, and (2) perform single bunch multiple extraction (SBME) at $25 \mathrm{~ms}$ intervals up to 12 times per AGS cycle. The remaining bunches, if any, have to be debunched and go though the slow extraction beam (SEB) channel for AGS HEP experiments.

With the NewFEB system the AGS will also serve as an injector for the RHIC, which is under construction. The circumference of the RHIC ring is 19/4 times larger than the AGS and its harmonic number at injection is 342 compared to 12 of the AGS. The present RHIC design assumes that the AGS will accelerate three bunches per pulse and transfer individual bunches one by one into the waiting if buckets in RHIC (SBME). Each RHIC ring will be filled with 57/114 bunches one after another in a few minutes every 10 hours or so and accelerate heavy ions to energies of $250 \cdot(\mathrm{Z} / \mathrm{A}) \mathrm{GeV} / \mathrm{N}$.

The schematic layout of the AGS-Booster complex is shown in Figure 1.

\footnotetext{
*Work performed under the auspices of the U.S. Department of Energy.
}

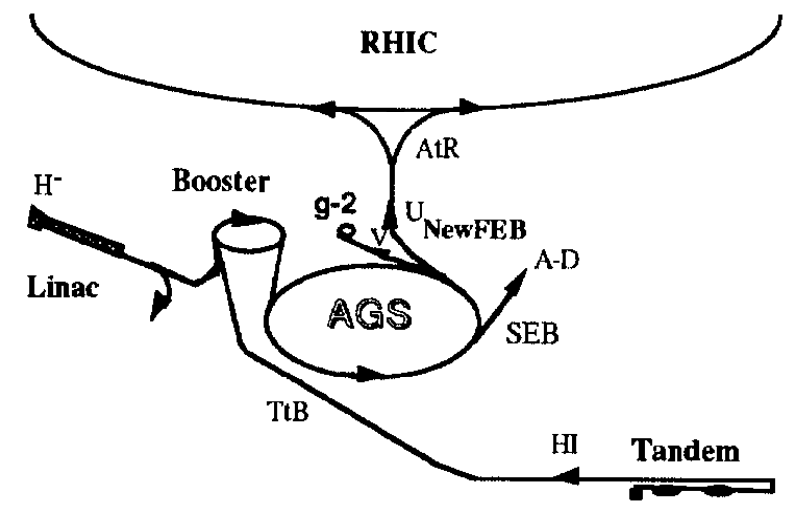

Figure 1. Schematic view of the AGS-Booster complex.

\section{DESIGN OF THE NewFEB}

\section{A. Machine and Beam Parameters}

Due to its high intensity operation for the $\mathrm{g}-2$ experiment and the neutrino experiment, it is important that the NewFEB system can achieve a high extraction efficiency $(>99 \%)$. On the other hand, for RHIC injection, the beam intensity is low but pulse-to-pulse and cycle-to-cycle modulations in the extracted bunch beam parameters must remain within acceptable levels since any excess will directly influence RHIC performance. Therefore, stability and reproducibility of the extracted beam parameters are crucial for RHIC injection.

For design purposes, we may assume that the operational NewFEB proton momentum range is (1) $22<\mathrm{p}<30 \mathrm{GeV} / \mathrm{c}$, (2) the $95 \%$ normalized transverse emittance of the high intensity beam should be $\varepsilon^{n_{h, v}}(95 \%)=6 \cdot \sigma^{2} / \beta \cdot(p / m) \leq 50 \pi$ $\mathrm{mm}$-mrad, where $\sigma$ is the standard deviation of the beam size due to the transverse emittance, and (3) the maximum total momentum spread allowed is $(\mathrm{dp} / \mathrm{p})_{\text {full }} \leq \pm 0.2 \%$. The actual measured values of $\varepsilon^{n}$ and $d p / p$ for the AGS beam are strongly dependent on the machine condition, especially the beam intensity. However, under the normal running conditions it is generally agreed that $\varepsilon^{n_{(95 \%)}}=(20-40) \pi \mathrm{mm}$-mrad, and $(\mathrm{dp} / \mathrm{p})_{\mathrm{full}}= \pm(0.05-0.12) \%$. The high intensity values with the Booster have not yet been established.

For RHIC injection, the expected values of $\varepsilon^{n}$ and dp/p for both protons and heavy ions are substantially lower than the current values since the AGS Booster can deliver much more intensity than that assumed for the RHIC design parameters.

It should be noted that a fast kicker and an ejector septum magnet must be located at straight section G10 and at straight section $\mathrm{H} 10$, respectively, in order to utilize the existing $U$ line and due to limited availability of straight sections. At 10foot straight section $(\mathrm{G} 10, \mathrm{H} 10), \beta_{h}$ and $\beta_{\mathrm{V}}$ are rapidly 
changing as well as $D_{\mathrm{X}}$. The parameters and performance of the present AGS machine are summarized as follows:

Table 1. AGS parameters.

\begin{tabular}{|c|c|}
\hline Circumference & $\mathrm{C}=2 \pi \mathrm{R}=807.075[\mathrm{~m}]$ \\
\hline Curvature & $\rho=85.17$ \\
\hline Revolution Time & $\mathrm{t}_{\mathrm{rev}}=2.692$ \\
\hline Tune & $\mathrm{Q}_{\mathrm{h}} \cong \mathrm{Q}_{\mathrm{V}} \cong 8.7$ \\
\hline Beta Functions & $\beta_{\mathrm{h}, \mathrm{v}}=22.5(10.5) \quad[\mathrm{m}]$ \\
\hline Dispersion Function & $\mathrm{D}_{\mathrm{x}} \max =2.20$ \\
\hline No. of Bunches & $\mathrm{N}_{\mathrm{b}}=12,(3$ for $\mathrm{HI})$ \\
\hline Full Bunch Length & $t_{b}=35 \pm 5$ \\
\hline Gap bet. Bunches & $t_{S}=224$ (peak-to-peak)[ns] \\
\hline Typical Intensity & $1.6 \cdot 10^{13}$ \\
\hline & $3.0 \cdot 10^{8}$ \\
\hline & $1.0 \cdot 10^{8}$ \\
\hline Typical AGS Cycle & 2.0 (FEB), 3.4 (SEB) [s] \\
\hline Typical Momentum & $\begin{aligned} \mathrm{p}= & 24.5,28.5(\mathrm{p})[\mathrm{GeV} / \mathrm{c}] \\
& 29.0 \cdot(\mathrm{Z} / \mathrm{A})[\mathrm{GeV} / \mathrm{c} / \mathrm{N}]\end{aligned}$ \\
\hline Emittance $\varepsilon^{\mathrm{n}_{\mathrm{h}, \mathrm{v}}(9}$ & $5 \%) \cong 35 \pi[\mathrm{mm}-\mathrm{mrad}](\mathrm{p})$ \\
\hline Momentum Spread & $(\mathrm{dp} / \mathrm{p})_{\mathrm{full}} \cong \pm 0.12 \quad[\%]$ \\
\hline
\end{tabular}

The following tables lists the expected beam parameters and performance of the AGS Complex for its NewFEB operation:

Table 2 . NewFEB beam parameters.

\begin{tabular}{|c|c|c|c|c|}
\hline Uots: & 96 & \multicolumn{3}{|c|}{ BHC BHIO } \\
\hline Particles $=$ & $\begin{array}{l}\text { Protons } \\
22-29\end{array}$ & \multicolumn{3}{|c|}{ Protons Heavy ions } \\
\hline & & \multicolumn{3}{|c|}{$\begin{array}{l}29.0 \\
\left(29.75 \cdot(\mathrm{Z} / \mathrm{A}) \text { for } 197 \mathrm{Au}^{77+}\right)\end{array}$} \\
\hline $\mathrm{N}_{\mathrm{SBE}}=$ & $1,3,(12)$ & \multicolumn{3}{|c|}{$3(19 \cdot 2(\cdot 2))$ for 2 rings } \\
\hline $\mathrm{N}_{\mathrm{p}} /$ Bunch $=$ & $<4000$ & 100 & $1-6$ & {$\left[10^{9}\right]$} \\
\hline$\%)=$ & $<50 \pi$ & $<20 \pi$ & $<10$ & {$[\mathrm{~mm}$} \\
\hline (d & $<+0.2$ & $< \pm 0.06$ & $< \pm 0.10$ & \\
\hline Abur & 1.0 & 0.3 & 0 & {$[\mathrm{el}$} \\
\hline $\mathrm{L}_{\text {bunch }}$ & $<40$ & $<17$ & $<17$ & {$[\mathrm{~ns}]$} \\
\hline
\end{tabular}

\section{B. Extraction Scheme}

The new system will consist of a fast multi-pulsing kicker at s.s.G10 followed by a thick septum ejector magnet at s.s.H10. To minimize the required voltage on pulsing the fast kicker we designed a C-type open ferrite magnet with a pole tip. The kicker will be placed about $50 \mathrm{~mm}$ from the central orbit. A few $\mathrm{ms}$ before the extraction two extraction bumps are created to bring the beam in to the aperture of the kicker and adjacent to the septum of the ejector. At extraction, the kicker is triggered every $25 \mathrm{~ms}$ to send one bunch at a time into the ejector, which gives an additional kick to extract the bunch out of the ring. In Figure 2, we show a schematic layout of the NewFEB extraction components and the particle trajectories.

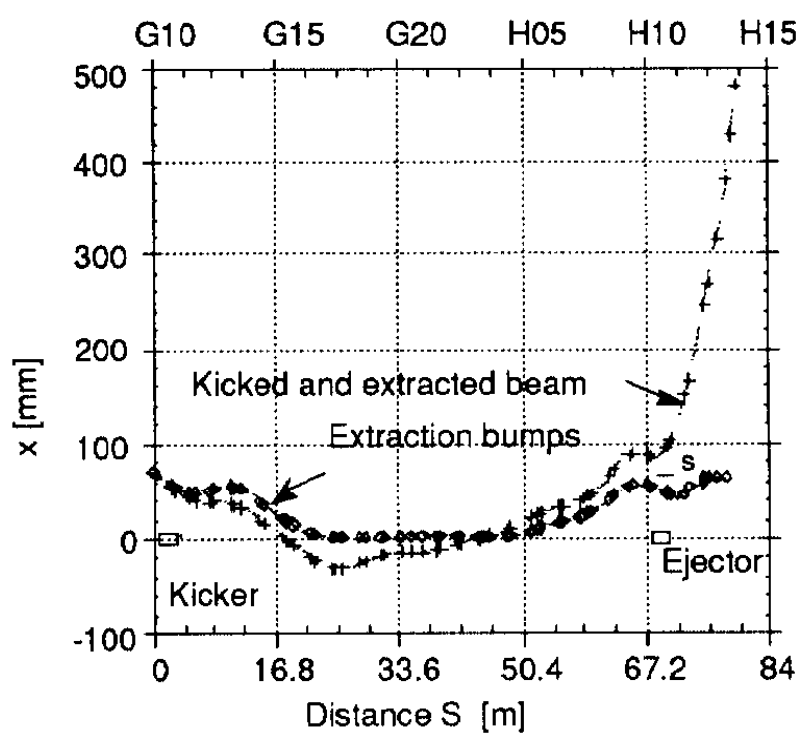

Figure 2. Layout of the NewFEB components.

\section{Fast Kicker, Ejector and Orbit Bumps}

Using the $99 \%$ emittance at $\mathrm{p}=29 \mathrm{GeV} / \mathrm{c}$, we have the full horizontal beam width $w_{\mathrm{f}} \leq 16.4 \mathrm{~mm}$ at both $\mathrm{G} 10$ and $\mathrm{H} 10$. Assuming that we need $2 \mathrm{~mm}$ clearance at both sides of the septum of the ejector magnet, and $10 \mathrm{~mm}$ septum thickness, then the required separation of the circulating beam and the beam kicked by the fast kicker is $\Delta x=30.4 \mathrm{~mm}$ at $\mathrm{H10}$. The kicker must deflect the beam by

$$
\theta(\mathrm{G} 10)=\Delta \mathrm{x} / \sqrt{\beta(G 10) \cdot \beta(H 10)} \cdot \sin (\Delta \mu)=-1.80 \mathrm{mrad},
$$

where $\Delta \mu$ is the betatron phase advance from the kicker to the septum calculated from the AGS lattice. This corresponds to $\int \mathrm{Bdl}=\mathrm{B}_{\mathrm{O}} \cdot \mathrm{l}_{\mathrm{eff}}=-0.18 \mathrm{~T}-\mathrm{m}$. Since it is desirable to keep the maximum pulse voltage less than $40 \mathrm{kV}$, the kicker magnet will be subdivided into four modules and powered in parallel. The kicker has a limited aperture, $32 \mathrm{~mm}$ x $22 \mathrm{~mm}(\mathrm{w} \mathrm{x} \mathrm{g}$ ) and a pole tip which is shaped to maximize the good field region while keeping a gap as large as possible as shown in Figure 3.

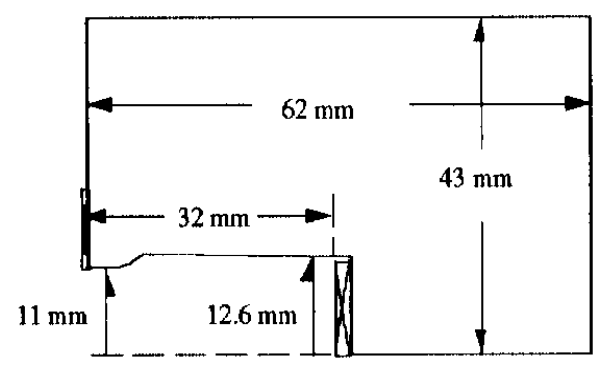

Figure 3. Geometry of the fast kicker.

For NewFEB operation, the ejector magnet also has to pulse every $25 \mathrm{~ms}$ up to 12 times per AGS cycle. As a result, the temperature of the copper septum will rise significantly, 
causing wear of the insulation. Therefore, the septum needs 10 $\mathrm{mm}$ thickness and must be water-cooled.

Two $1 \lambda$ rather than standard $3 / 2 \lambda$ local orbit deformations are created to avoid the kicked beam hitting the vacuum chamber wall around at s.s.G17. These bumps are generated by powering backleg windings on selected AGS main magnets so arranged that the tune shifts and stopbands at $\mathrm{Q}_{h}=8.5$ are minimized. The basic design parameters of the kicker, ejector and extraction bumps are summarized in Table 3 .

Table 3 . NewFEB magnet parameters.

\begin{tabular}{|llll|}
\hline & Kieker & Edector & Bumps \\
\hline Aperture[mm] & $32 \times 22$ & $72 \times 25$ & Full \\
$\theta_{\text {max }[\mathrm{mrad}]}$ & 2.0 & 22.0 & $2.0 /$ pair \\
wave form & half since & half sine & half sine \\
tbase & $360 \mathrm{~ns}$ & $2 \mathrm{~ms}$ & $5 \mathrm{~ms}$ \\
$I_{\max }$ & $2.0 \mathrm{kA}$ & $23 \mathrm{kA}$ & $1.0 \mathrm{kA}$ \\
$I_{\max }$ & $2.0 \mathrm{kA}$ & $23 \mathrm{kA}$ & $1.0 \mathrm{kA}$ \\
Tolerance & $<0.9 \%$ & $<0.09 \%$ & $<0.8 \%$ \\
\hline
\end{tabular}

\section{Beam Instrumentation}

In order to observe possible bunch-by-bunch beam scraping during the NewFEB extraction, the new fast beam loss monitors will be installed at key extraction points. The present beam position monitor (PUE) system will be upgraded with a universal bunch-by-bunch digitizer so that we can take orbit data at the if rate, allowing tracking over many orbits. It should also improve absolute beam position measurement capabilities.

\section{SIMULATION}

To find out the circulating and the extracted beam parameters at the middle of s.s.H13 (the entrance of the existing U-line), simulation studies are performed with a model of the AGS, using the accelerator modeling program MAD. We run MAD to obtain the desired orbit at the kicker and at the ejector, making adjustments of the extraction bumps at the tune $\mathrm{Q}_{\mathrm{h}} \equiv 8.75$. Then, the particle with initial conditions $\left\{x, x^{\prime}\right\}$ at the beginning of s.s.G10 is traced through the lattice and receives an appropriate kick (1.6 mrad) at the kicker and an additional kick ( $21 \mathrm{mrad}$ ) at the ejector up to the middle of s.s.H13, where the beam should be about $43 \mathrm{~cm}$ away from the central orbit, free from the fringe field of the ring magnets as shown in Figure 2.

The simulation results show that the optical parameters $\left.\left(\beta_{\mathrm{h}, \mathrm{v}}, \alpha_{\mathrm{h}, \mathrm{v}}, D_{\mathrm{X}}, D_{\mathrm{X}}\right)^{\prime}\right)$ at s.,s.H13 are sensitive to fine bump tunings and fringe field effects from the septum to s.s.H13. Therefore, tracking studies using the AGSBEAM code which has a complete main magnet field map [7] and TOSCA 3D program [8] are also being performed to confirm the MAD results at s.S.H13 for the proper optical design for the U-line and its optical matching with the AtR line.

\section{CONCLUSIONS AND OUTLOOK}

The basic engineering design is made on the NewFEB system at the AGS, which is capable of performing single bunch multiple extraction every $25 \mathrm{~ms}$ up to 12 times per AGS cycle for $\mathrm{g}-2$ experiment and RHIC injection. It is expected that the g-2 muon storage ring and RHIC are expected to be completed in 1994 and in 1997, respectively. Due to its high intensity operation for the $\mathrm{g}-2$ experiment, it is important that the NewFEB system can achieve a high extraction efficiency. On the other hand, for RHIC injection, stability and reproducibility of the extracted bunched beam parameters are crucial since any change of the extracted beam parameters will directly influence RHIC performance. Further simulation studies of the NewFEB extraction and machine studies with the Booster will be needed to specify the NewFEB extraction performance as well as the overall required AGS capability as the injector for RHIC and the high intensity proton machine.

\section{ACKNOWLEDGMENTS}

We would like to thank many members of the AGS Department who have made contributions to this project, especially: E. Rodger (septum), A. Soukas and W. Zhang (power supplies, PFN), J. Tuozzolo and W. Meng (kicker) and R. Witkover (instrumentation). We are also grateful to E. Bleser, H. Brown, H.W. Foelsche, E. B. Forsyth, J.W. Glenn, A. McNerney, W.T. Weng for their helpful discussions and suggestions received during our design work on the NewFEB system.

\section{REFERENCES}

[1] W.T. Weng, "The AGS New Fast Extraction System and the Single Bunch Extraction", IEEE Trans. Nucl. Sci. NS30, No. 3 (1983).

[2] M. Tanaka and Y.Y. Lee, "The AGS New Fast Extraction System for the g-2 Experiment and RHIC Injection", 1991 IEEE Part. Accel. Conf. (Sn. Francisco), p. 9589.

[3] AGS Experiment 821, "A New Precision Measurement of the Muon g-2 Value at the Level of $0.35 \mathrm{ppm}$ ".

[4] BNL-52195, "Conceptual Design of Relativistic Heavy Ion Collider", May 1989.

[5] AGS Experiment 889, "Proposal for a Long Baseline Neutrino Oscillation Experiment at the AGS".

[6] W.T. Weng, "Operation of the Brookhaven AGS with the Booster", this conference.

[7] H.W. Foelsche and N. Tsoupas, private communication.

[8] W. Meng, private communication. 